\title{
De la pensée latérale
}

\section{Eberhard Wolff}

Prof. Dr rer. soc., rédacteur culture, histoire, société

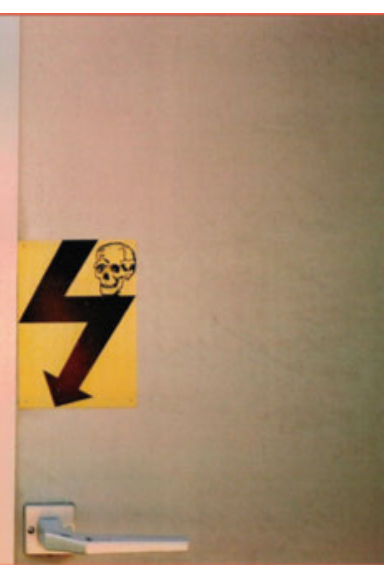

«Si l'on ouvrait la porte - et la poignée laisse présager une ouverture vers l'intérieur - et à condition qu'elle puisse être ouverte, on entrerait dans le royaume des morts. Le crâne représenté à côté de l'éclair noir ne souffre aucune autre interprétation. C'est comme cela, de cette manière si froide et prosaïque, qu'il faut imaginer l'entrée du 'monde d'en dessous', de l'outre-tombe. Là règne indubitablement la mort. Un peu décevante cette entrée, malgré tout l'étonnement que suscite l'hégémonie permanente, omniprésente, universelle, dominante des maîtres des morts. Et il y a une certaine cohérence entre le côté simple de la porte et le fait que depuis des millénaires, les gardiens des enfers n'aient pas trouvé d'autre emblème que leurs crânes. Et pourtant, l'idée qu'à l'extrême, il n'y a que quelques millimètres entre l'au-delà et nous, entre ici et le néant est séduisante.»

Les lecteurs assidus de cette revue devineront bien vite qui explique la photo présentée ici: Enrico Danieli. Depuis de nombreuses années, le médecin et écrivain du Tessin marque ce journal de sa patte particulière. Récemment par exemple dans son article sur sa confusion entre sexe, titre et nom des médecins ou sa déprimante humiliation en tant que patient sous lithium.

Dans son ouvrage tout juste paru [1], il s'est penché sur le phénomène des panneaux. Ces dernières années, lors de ses tournées, il les a immortalisés avec son appareil photo privé. Panneaux indicateurs, d'information, de danger ou d'interdiction. Et il a complété 66 d'entre eux de "pirouettes linguistiques». C'est de l'une de celles-ci que provient l'entrée de l'au-delà susmentionnée.

Bien entendu, le livre inclut aussi des écriteaux bricolés maison, dont le texte maladroit ou inhabituel s'avère involontairement comique: "Ouverture pour petits animaux (pour incinération)» ou «Corps d'animaux. Têtes de vaches entières ou autres déchets d'abattage pour incinération». Danieli ne s'arrête pas là. Il a pour habitude de comprendre les choses de travers. Voilà comment la banale porte du poste haute tension se transforme en entrée des enfers. Une flèche malencontreusement dirigée vers le haut sur un panneau «Privé» est selon Danieli la preuve qu'au ciel aussi, il y a des propriétaires privés. Ce n'est pas mé- chanceté de sa part. Cela lui permet d'entrer dans de nouvelles dimensions de pensée.

L'auteur a un faible évident pour les panneaux indiquant les toilettes. Surtout quand ils voisinent avec des panneaux vers d'autres destinations, avec lesquels ils sont mis sur un pied d'égalité: ici, on renvoie à la merveille naturelle des marmites du diable... et aux W.-C. Deux indicateurs jumeaux sur fond vert. Mais si les panneaux verts annoncent des réserves naturelles, l'écriteau «W.-C.» vert guide-t-il vers des toilettes biologiques? Une marmite naturelle?

Ici, on signale une église... et les W.-C. Cette fois sur fond bleu. L'église pour soulager le fardeau de l'âme. Les W.-C. pour soulager le fardeau de la vessie. Et tout à coup, de nombreux points communs entre le petit coin et le lieu saint sautent aux yeux.

Changement de décor avec un funérarium. Une inscription sur une porte: vers les salles d'exposition - et bien sûr les W.-C. "Le lien est sans doute l'élimination, les morts de la société, les déchets de l'organisme.»

La pensée latérale a quelque chose d'anarchique. Et elle mène aux limites de la vie, sur lesquelles Danieli travaille, avec lesquelles il flirte. La noyade ponctue comme un fil rouge son "Divertimento a bassa voce», accompagnée de l'abattage, de l'empoisonnement, du meurtre, de l'enfer, de la mort, de la fin. Une photo montre une bouée qui tangue sur les vagues, à distance de la plage. Elle met en garde les nageurs insouciants avec l'inscription "Pourrez-vous revenir?». "S'il n'est pas possible de revenir», rétorque Danieli, «on peut aller plus loin, toujours plus loin.»

Oui, l'idée d'ouvrir la porte avec la tête de mort et l'éclair et de regarder à l'intérieur peut être tentante. Il n'empêche que je ne déconseillerais pas à seulement mon collègue Danieli de le faire pour de vrai. Il pourrait bien ne rien y avoir derrière l'entrée du royaume des morts. Ou juste un poste haute tension barbant.

\section{Crédit photo}

Enrico Danieli

\section{Référence}

1 Danieli E. 66 Plaquetten mit Pirouetten. Bagatellen zu Pictogrammen, Photogrammen, Paragrammen und ihren Melodram men. Literareon, 166 p., 29 €, 39 CHF. ISBN 978-3-8316-2024-1. 